Protocoll der Vorstands-Sitzung rom 31. Juli 1878.

Anwesend die Herren: C. Liebermann, R. Biedermann, A. Franck, A. Geyger, P. W. Hofmann, A. Holtz, G. Krämer, C. A. Martius, C. Scheibler, E. Scbering, Eug. Sell, F. Tiemann.

1) Die in der Sitzung rom 11. Mai als Commissäre des Vorstandes der Gesellschaft bestellten Herren: E. Schering und F. Tiemann berichten über die Resultate der Verhandlungen, welche sie behufs Anbahnung eines günstigeren Verlagsverhältnisses der Berichte sowobl mit der $D$ ümmler'schen als mit verschiedenen anderen Buchhandlungen gepflogen haben. Der Vorstand beschliesst darauf endgültig die Berichte selbst zu verlegen und die Herren R. Friedländer und Sohn als seine buchbändlerischen Commissäre zu bestellen. Es wird eine, aus den Herren E. Schering, F. Tiemann und H. Wichelh a u $s$ bestehende Commission gewählt, welcher die Ausarbeitung eines detaillirten Contractes mit der genannten Buchhandlung mit der Verplichtung übertragen wird, den Entwurf so bald wie möglich dem Vorstande zu definitiver Genehmigung vorzulegen.

2) Das Bureau wird beauftragt, den Director der Berg-A kademie, Herrn Bergrath $\mathrm{Hau}$ checorne, im Namen des Vorstandes schriftlich um Erlaubniss zu bitten, die Sitzungen der Gesellschaft vom 14. October $1878 \mathrm{ab}$ im Saale des neuen Institutes der Berg-Akademie (Invaliden-Strasse No. 45) abhalten zu dürfen.

3) Der Redacteur wird ersucht, in Zukunft in dem letzten vor den grossen Ferien erscheinenden Hefte der Berichte den Termin zu veröffentlichen, bis zu welchem Arbeiten für das Ferienheft bei der Redaction eingeliefert werden müssen.
Der Schriftführer:
Der Vorsitzende:

Ferd. Tiemann.

C. Li e b e rmann.

\title{
Mittheilungen.
}

449. E. Demole: Ueberführung des Dibromäthylens in ein Keton mit 4 A tomen Kohlenstoff mittelst unterbromiger Säure.

(Eingegangen am 8. October; verl. in der Sitzung von Hrn. A. Pinver.)

Die letzthin ron mir vorgeschlagene Theorie ${ }^{1}$ ) zur Erklärung der Oxydation und Polymerisation des Dibromäthylens durch freien Sauerstoff setzt die vorübergehende Bildung eines ungesättigten Körpers $\mathrm{C}_{2} \mathrm{HBr}$ roraus. Letzterer würde sich unter Wiederaufnahme

1) Diese Berichte XI, 1307 . 
ron $\mathrm{HBr}$ bald oxydiren und in Bromacetylbromid verwandeln, bald aber polymerisiren und $\mathrm{zwar}$ ebenfalls unter Wiederaufnahme von $\mathrm{HBr}$ je nach der Temperatur und dem Gehalte dẹs umgebenden Mittels an Sauerstoff.

Um dieser Anschauungsweise irgend welchen Anhaltspunkt zu rerschaffen, erachtete ich es für nothwendig die ungesättigte Verbindung zu isoliren $z u$ suchen oder dieselbe doch wenigstens in eine beständige Verbindung äberzuführen, um sich über deren Existenz nicht täuschen zu können.

Die unterbromige Säure besitzt bei nicht sehr hoher Temperatur die doppelte Eigenschaft, nämlich einerseits eine Quelle nascirenden Sauerstoffs zu sein und andererseits sich leicht mit ungesättigten Molekülen zu rerbinden. Von diesem Gesichtspunkte aus liess sich erwarten, dass bei einer nicht sehr hohen Temperatur diese Säure in Berührung mit Dibromäthylen letzterem Sauerstoff abgeben würde, and dass der Uebergangskörper $\mathrm{C}_{2} \mathrm{H} \mathrm{Br}$, entstanden in Folge dieser Oxydation, sich mit 1 oder 2 Molekülen derselben unterbromigen Säure sättigen würde.

Der Versuch hat diese Voraussetzungen theilweise bestätigt, hat aber ferner auch noch zu unerwarteten Thatsachen geführt. In Folgendem werden die bezüglichen Details der Operationen mitgetheilt werden.

Einwirkung der unterbromigen Säure auf Dibromäthylen.

Reinem und frisch bereitetem Dibromätbylen wurde eine abgekühlte 5.3 procentige wässerige Lösung unterbromiger Säure im Verhältaiss von $11 \mathrm{ccm}$ auf $1 \mathrm{~g}$ Dibromäthylen zugefügt.

Die so erhaltene Mischung wurde während 1 Stunde tüebtig umgeschüttelt, wobei eine fühlbare 'Temperaturerhöhung beobachtet warde ${ }^{1}$ ).

Nach Verlauf dieser Zeit ist die Reaction beendet und man beobachtet alsdann eine Volumverminderung des am Boden des Glaskolbens befindlichen Dibromäthylens, und ferner, dass die ganze Flüssigkeitsmasse durch Brom eine gelbliche Färbung angenommen hat, was uns einen sichern Beweis liefert, dass dieselbe keinen ungesättigten Körper mehr entbält. Sowohl die überstehende Flüssigkeit als auch die ölige Schicht wurden nun getrennt untersucht.

\section{A. Flüssigkeit.}

Dieselbe wurde mit Schwefelwasserstoff, jedoch unter Vermeidung eines Ueberschusses, behandelt, um das Quecksilberbromid abzuscheiden. Nachdem das $\mathrm{HgS}$ durch Filtration von der Flüssigkeit getrennt

1) Bei einer Flussigkeitsmenge von $1500 \mathrm{ccm}$ mit einer Anfangstemperatur von $13^{\circ} \mathrm{C}$. wurde nach einigen Minuten langem Schutteln eine Temperaturerhöhung von $20^{\circ} \mathrm{C}$. beobachtet und war das Thermometer somit auf $33^{\circ} \mathrm{C}$. gestiegen. Letztero Temperatur schien indessen nicht uberstiegen worden zu sein. 
worden, wurde letztere mit Aether behandelt und derselbe dann der Destillation unterworfen. Der Aether hinterliess einen Rückstand, der eine zwischen $200-210^{\circ} \mathrm{C}$. destillirbare Säure liefert, deren Analysen mit der Formel der Bromessigsäure übereinstimmen. Die Bildung dieser Säure lässt sich leicht erklären sobald man sich erinnert, dass sich das Dibromäthylen unter dem Einfluss von Sauerstoff in Bromacetylbromid verwandelt und dieses durch Wasser in Bromwasserstoff und Bromessigsäure $\mathrm{C}_{2} \mathrm{H}_{3} \mathrm{BrO}_{2}$ umgesetzt wird.

B. Oelige Flüssigkeit.

Dieselbe stellt eine schwere, klebrige, nach Brom riechende Masse dar. Auf den ersten Augenblick würde man sie für das Bromadditionsprodukt des $\mathrm{C}_{2} \mathrm{H}_{2} \mathrm{Br}_{2}$ mit $\mathrm{Br}_{2}$ halten. Vor Luftzutritt geschützt, scheint dieser Körper keine Veränderung zu erleiden, aber sobald derselbe in dünuer Schicht auf eine Glasscheibe gebracht wird, fängt er bald an zu krystallisiren in Folge Abdunstung eines flüchtigeren Bestandtheiles ${ }^{1}$ ).

Zur Beschleunigung dieser Trennung wurde das rohe Oel anf einem grossen Ührglase während 4-5 Stunden auf dem Wasserbade erhitzt und nach dieser Zeit blieb beim Erkalten eine braune, feste, krystallinische Masse zurück. Auf leichte und vollständige Weise wird die Reinigung dieses Körpers erzielt, wenn man ihn zwischen porösen Thonplatten presst und dann 2-3 Mal aus starkem, heissen Alkohol umkrystallisirt. Auf diese Weise erbält man ihn in grossen prismatischen, büschelweise gruppirten Krystallen, deren Schmelzpunkt $\mathrm{z}$ wischen $89-90^{\circ} \mathrm{C}$. liegt und die bei einer $100^{\circ} \mathrm{C}$. etwas übersteigenden Temperatur zersetzt werden. Was seine Löslichkeitsverhältnisse betrifft, so gestalten sich dieselben folgendermaassen: In kaltem Wasser ist er beinahe rollständig unlöslich, ron kaltem Alkohol wird er wenig gelöst, dagegen löst er sich in ziemlicher Menge in starkem, kochenden Alkohol, in Aether, sowie in kochendem Methyl- und Amylalkohol. Der geschmolzene Körper besitzt bei $0^{0}$ das spec. Gew. ron 2.88 .

Eine beträchtliche Zahl mit einander übereinstimmender Analysen fübren zu der Formel $\mathrm{C}_{4} \mathrm{H}_{2} \mathrm{Br}_{6} \mathrm{O}$, obgleich die für den Wasserstoff gefundenen Zablen etwas zu hoch sind.

Von Brom and Säuren wird dieser Körper sogar in der Wärme nicht merklich angegriffen; heisse alkoholische Kalilösung scheint nicht rasch zerstörend darauf einzuwirken. Nach 5 stündigem Erhitzen auf $150^{\circ}$ in geschlossener Röhre mit Acetylchlorid wurde beim Oeffnen derselben keine Chlorwasserstoffentwicklung beobachtet und

1) Fin Theil des roben Oeles wurde destillirt, aber ther $100^{\circ}$ erhitzt fangt es an sich zu zersetzen; bei $205^{\circ}$ geht eine unter Zersetzung destillirende Flüssigkeit aber, deren Analysen der Formel $\mathrm{C}_{2} \mathrm{H}_{2} \mathrm{Br}_{4}$ entsprechen. 
der umkrystallisirte Inbalt ergab bei der Analyse immer wieder der Formel $\mathrm{C}_{4} \mathrm{H}_{2} \mathrm{Br}_{6} \mathrm{O}$ entsprechende Zahlen. Essigsäureanhydrid hat unter ähnlichen Bedingungen ebenfalls keine Veränderungen des Körpers hervorgerufen.

Aus diesem Verbalten muss angenommen werden, dass der betreffende Körper, obgleich sauerstoff baltig, keine Hydroxylgruppe enthalte, und dass daher der Sauerstoff 2 Valenzen des Kohlenstoffes binde. Der Körper ist daher ein Aldehyd, Keton oder Alkyloxyd und seine Constitation wurde mittelst Oxydation und Reduction zu ermitteln gesucht.

\section{Reduction der Verbindung $\mathrm{C}_{4} \mathrm{H}_{2} \mathrm{Br}_{6} \mathrm{O}$.}

$Z_{\mathrm{i}}$ diesem $Z$ wecke wurde ein langsamer Salzsäurestrom durch eine heisse, alkoholische Lösung des Körpers, in welcher sich Natriumamalgam ( $5 \mathrm{pCt}$.) befand, geleitet. Nach Verlauf von $1-2$ Stunden ist die Reaction beendigt und die saure Flüssigkeit wurde hierauf mit Natriumcarbonat neutralisirt, filtrirt und unter Umschütteln mit einer conc. Natriumbisulfitlösung versetzt. Nach 1 stündigem Contakt wurden die Krystalle getrennt, mit Aether ausgewaschen, getrocknet und dann mit ihrem Volumen Natriumcarbonat und Wasser versetzt. Das Ganze wurde nun der Destillation unterworfen, wobei ein mit viel Wasser gemischter Körper, der flüchtiger als dieses ist, erhalten wurde. Das so erbaltene wässerige Destillat wurde mit Aether bebandelt, letzterer dann über Kaliumcarbonat getrocknet und nachher destillirt. Nach dem Uebergange des Aethers wurde eine kleine Menge eines gegen $79-81^{\circ}$ C. siedenden Körpers, der mit gewöhnlichem Alkohol keinerlei Aehnlichkeit besitzt, und dessen Analysen mit der Formel $\mathrm{C}_{4} \mathrm{H}_{8} \mathrm{O}$ derjenigen des Aethylmethylketons, übereinstimmen, erhalten. Mit Natriumbisulfit gibt der Körper eine krystallinische Verbindung, die nach rorheriger Reinigung, bei der Analyse die der Formel $\mathrm{C}_{4} \mathrm{H}_{9} \mathrm{NaSO}_{4}$ entsprechende Zahlen liefert. Diesen Versuchen gemäss kann man annehmen, dass der der Reduction unterworfene Körper $\mathrm{C}_{4} \mathrm{H}_{2} \mathrm{Br}_{6} \mathrm{O}$ nichts anderes ist als ein Keton, nämlich Hexabromätbylmethylketon.

\section{Oxydation des Hexabromäthylmetbylketons.}

Zur besseren Aufklärung der Constitution dieses Körpers und zur Aufhellung einer interessanten Frage wurde derselbe der Oxydation unterworfen. Es handelte sicb nämlich darum zu erfahren, welches die Oxydationsprodukte sein würden, im Falle die 6 Bromatome nur an die 2 endständigen Koblenstoffatome der Kette gebunden wären, nämlich $\mathrm{C} \mathrm{Br}_{3} \ldots \mathrm{C}=:=\mathrm{O} \ldots \mathrm{CH}_{2} \ldots \mathrm{CBr}_{3}$.

Wenn das Popoff'sche Gesetz für die bromirten Ketone ebenfalls gültig ist, so würde man durch Oxydation eine zweibasische Säure, 
die Malonsăure neben Kohlensäure erhalten, dagegen Mesoxalsăure und Kohlensäure, wenn sich die 6 Bromatome auf 3 Koblenstoffatome vertheilen, nämlich $\mathrm{CHBr} \mathrm{Br}_{2} \ldots \mathrm{C}==\mathrm{O} \ldots \mathrm{CBr}_{2} \ldots \mathrm{CHBr} \mathrm{Br}_{2}$.

Rauchende Salpetersäure greift bei der Wasserbadtemperatur die Verbindung $\mathrm{C}_{4} \mathrm{H}_{2} \mathrm{Br}_{6} \mathrm{O}$ heftig an und nach und nach rerschwindet diese unter stromweiser Entwicklung von Dämpfen. Letztere wurden nach vorherigem Waschen mit Wasser in ganz klares Barytwasser geleitet und schon nach ganz kurzer Zeit trübte sich dieses in Folge der Bildung von Bariumcarbonat, was entschieden die Entstehung von $\mathrm{CO}_{2}$ beweist. Die Oxydation ist beendet, sobald die Bromverbindung verschwunden und beim Erkalten der salpetersauren Lösung nichts mehr auskrystallioirt. Die salpetersaure Lösung wurde zur Trockne abgedampft, der Rückstand mit Wasser bebandelt und 2-3 Mal umkrystallisirt. Auf diese Weise erbält man blättrige Krystalle, die kein Brom enthalten, in Wasser und Alkohol löslich und sehr sauer sind, und deren Schmelzpunkt bei $139-140^{\circ} \mathrm{C}$. liegt. Die wässerige Lösung der Säure ist durch Chlorcalcium nicht fällbar. Die bei $100^{\circ} \mathrm{C}$. getrocknete und analysirte Substanz fübrt zu Zahlen, die der Formel $\mathrm{C}_{3} \mathrm{H}_{4} \mathrm{O}_{4}$, derjenigen der Malonsäure, entsprechen. Die wässerige Lösung der Säure mit Barythydratlöвung versetzt und der Ueberschuss der letzteren mittelst Kohlensäure entfernt, hinterlässt beim Abdampfen eine krystallinische Kruste, welcher die Formel $\mathrm{C}_{3} \mathrm{H}_{2} \mathrm{BaO}_{4}$ zukömmt. Dieses Salz, das sich in Wasser und selbst in heissem wenig löst, ist kein anderer Körper als das neutrale Bariummalonat. Aus diesen Thatsachen ergibt es sich mithin, dass im Hexabromäthylmethyketon, erbalten mittelst $\mathrm{C}_{2} \mathrm{H}_{2} \mathrm{Br}_{2}$ und $\mathrm{BrOH}$, die 6 Bromatome in gleicher Weise auf die 2 endständigen Kohlenstoffatome der Kette vertbeilt sind, und dass die Constitution des betreffenden Ketons $\mathrm{CBr}_{3} \ldots \mathrm{C}=:=\mathrm{O} \cdots \mathrm{CH}_{2} \ldots \mathrm{CBr}_{3}$ ist. Ferner findet bei der Oxydation dieser Verbindung die Spaltung nach dem Popoff'schen Gesetze statt, aber wegen der Gruppen $\mathrm{CBr}_{3}$ entsteht $\mathrm{CO}_{2}$ und eine zweibasische Säure, die Malonsäure.

\section{Seblussfolgerungen.}

Aus Gesagtem scheint es festgestellt za sein, dass sich bei der Oxydation des Dibromäthylens, wie ich es vermuthete ${ }^{1}$ ), wirklich ein Uebergangskörper $\mathrm{C}_{2} \mathrm{H}$ Br, Bromacetylen, im status nascendi oder ein Isomerer bildet. Bei niederer Temperatur polymerisirt sich dieser Körper unter Wiederaufnahme von $\mathrm{HBr}$, wäbrend er bei höberer Temperatur zugleich Sauerstoff und Bromwasserstoff aufnimmt und in Bromacetylbromid übergeht. In Gegenwart von $\mathrm{BrOH}$ verdoppelt sich sein Molekül, sättigt sich mit $\mathrm{HBr}$ und $\mathrm{Br}$ und bildet so den

1) Diese Berichte XI, 1307. 
sekundären Hexabrombatylalkohol, wie es folgende Gleichungen veranschaulichen.

1) $2\left(\mathrm{C}_{2} \mathrm{HBr}\right)+\mathrm{BrOH}=\mathrm{CBr}_{2} \ldots \mathrm{CHOH} \cdots \mathrm{CH} \cdots \mathrm{CBr}$;

2) $\mathrm{CBr}_{2} \ldots \mathrm{CHOH} \ldots \mathrm{CH}-. \mathrm{CBr}+\mathrm{HBr}+\mathrm{Br}_{2}$

$$
=\mathrm{CBr}_{3} \ldots \mathrm{CHOH}-. \mathrm{CH}_{2} \ldots \mathrm{CBr}_{3} \text {. }
$$

Die Gruppe $\mathrm{CH} . \mathrm{OH}$ kann wahrscheinlich nicht als solche in der Nähe von so vielen Bromatomen existiren und wandelt sich in die Gruppe $\mathrm{C}::=\mathrm{O}$ um, wodurch Hexabromätbylmethylketon ent steht. Die Leichtigkeit, mit der sich dieser Uebergangskörper polymerisirt und oxydirt ist bemerkenswerth. Freier Sauerstoff bildet damit den Typus Aldehyd, nascirender Sauerstoff und $\mathrm{BrOH}$ den Typus Keton unter Verdopplung seines Moleküles. Vielleicht wird es gelingen zum Typus Aethylenoxyd unter veränderten Bedingungen, was übrigens diese Untersuchungen bezwecken, zu gelangen.

Vevey, 6. October 1878.

450. F. Beilstein: Ueber die Scheidung des Zinks vom Nickel.

(Vorgetragen vom Verfasser in der Sitzung vom 27. Mai 1878; eingegangen am 5. October.)

Wöhler's Verfahren, nach Zusatz von Kali und Blausäure das Zink durch Einfach-Schwefelkalium zu fällen, ist bis jetzt das einzige exacte Verfahren zur Trennung des Zinks rom Nickel. Demselben haften leider mehrere Uebelstände an. Das aus alkalischer Lösung gefällte Schwefelzink verstopft ausserordentlich die Poren des Filters und ist mühsam zu waschen. Es ist nothwendig, freie Blausäure anzuwenden oder eine frisch bereitete Lösung chemisch reinen Cyankaliums. Eine Lösung von Cyankalium, die nur einige Zeit gestanden hat, ist ganz unbrauchbar (Klaye und Deus, Zeitschr. f. analytische Chemie 10, 197). Endlich bat man in Filtrat vom Schwefelzink zunächst das äberschüssige Cyankalium zu zerstören, ehe das Nickel gefällt werden kann.

Diese Umstände veranlassten bereits K laye und $D_{\text {e u }}{ }^{1}$ ), sich nach einer anderen Scheidungsmethode umzusehen, und sie fanden, dass Brunner's Verfahren ${ }^{2}$ ), richtig ausgeführt, durchaus befriedigende Resultate giebt. Danach wird die stark verdünnte Lösung der Chloride oder Nitrate ( $1 \mathrm{~g}$ Oxyde in wenigstens $500 \mathrm{ccm}$ Wasser gelöst) mit Soda bis zur Entstehung eines bleibenden Niederschlags versetzt und letzterer durch einen Tropfen Salzsäure gelöst. Man leitet nun Schwefelwasserstoff ein und sobald der Niederschlag ron Schwefel-

1) Zeitschr. f. analyt. Chemie 10, 190.

$\left.{ }^{2}\right)$ Jahresb. f. Chemie 1858, 621 . 